\title{
Vaccine Hesitancy and Exposure to Misinformation: a Survey Analysis
}

\author{
Stephen R. Neely, $P h D^{\top}$ (D), Christina Eldredge, $M D, P h D^{2}$, Robin Ersing, $P h D^{7}$, and \\ Christa Remington, $\mathrm{PhD}^{7}$
}

'School of Public Affairs, University of South Florida, 4202 E. Fowler Ave, SOC 107, Tampa, FL, USA; ${ }^{2}$ School of Information, University of South Florida, 4202 E. Fowler Ave, CIS 1040, Tampa, FL, USA.

INTRODUCTION: Despite the widespread availability of SARS-CoV-2 vaccines in the USA, vaccine hesitancy continues to represent a significant impediment to the attainment of herd immunity and the end of the COVID-19 pandemic. This survey analysis provides an update for clinical healthcare providers and public health officials regarding current trends in misinformation exposure, as well as common objections to COVID-19 vaccination.

METHODS: We conducted a web-based survey of 600 adults in the state of Florida between June 3 and June 14, 2021. Access to the sample was purchased through an industry-leading market research provider (Prodege MR), and survey respondents were selected using a stratified, quota sampling approach to ensure representativeness. Balanced quotas were determined (by region of the state) for gender, age, race, education, and ethnicity. The survey responses were analyzed using basic descriptive statistics, as well as chi-square testing and a logit regression model.

FINDINGS: High levels of misinformation exposure were observed among participants, with $73 \%$ reporting some exposure to misinformation about COVID-19 vaccines in the past 6 months. Exposure to misinformation was directly correlated with vaccine hesitancy. Among those who did not report any exposure to misinformation, $73.8 \%$ of respondents were vaccinated. That number fell to $62.9 \%$ with exposure to just one misinformation theme and $52.2 \%$ for six or more $\left(\chi^{2}=11.349 ; \varphi=0.138 ; p \leq\right.$ 0.05). Politicization was also found to be a major factor in vaccine hesitancy, with $73.4 \%$ of self-identified Democrats being vaccinated, compared to only $58.5 \%$ of Republicans and 56.5\% of Independents $\left(\chi^{2}=16.334 ; \varphi=0.165\right.$; $p \leq 0.001)$. Both misinformation exposure and political affiliation were strong predictors of vaccination even after accounting for other demographic predictors.

DISCUSSION: The survey results add to previous research on misinformation and vaccine hesitancy by quantifying exposure to specific misinformation themes and identifying its relationship to vaccine hesitancy. Overcoming these impediments to vaccination will require strategic and targeted messaging on the part of public health professionals, which may be aided by collaboration with political thought leaders. Understanding the volume and nature of misinformation themes the public is exposed

Received July 11, 2021

Accepted September 24, 2021

Published online October 20, 2021 to regarding COVID-19 vaccines may aid public health officials in targeting this vaccine messaging to more directly address reasons for vaccine hesitancy.J Gen Intern Med

J Gen Intern Med 37(1):179-87

DOI: $10.1007 / \mathrm{s} 11606-021-07171-z$

(c) Society of General Internal Medicine 2021

\section{INTRODUCTION}

Despite the widespread availability of SARS-CoV-2 vaccines in the USA, vaccine hesitancy continues to represent a significant impediment to the attainment of herd immunity and the end of the COVID-19 pandemic. ${ }^{1,2}$ As of early June 2021, data provided by the Centers for Disease Control (CDC) showed that less than half of all eligible Americans $(48.6 \%)$ were "fully vaccinated". 3 Throughout the summer of 2021, the USA has struggled to keep pace with vaccination goals established by the Biden administration. ${ }^{4,5}$ Recent research suggests that a number of factors may contribute to this reluctance, including the politicization of the COVID-19 pandemic, concerns over the rapid development of the vaccine, and the continued spread of misinformation related to vaccine safety and efficacy. ${ }^{6,7}$ In particular, these studies suggest that the proliferation of misinformation about COVID-19 vaccines may be a significant driver of vaccine hesitancy, while conversely, exposure to reliable medical information may improve vaccine acceptance. $^{8-10}$

With these concerns in mind, it is critical for both clinical providers and public health officials to understand the factors underlying vaccine hesitancy, as well as the evolving misinformation landscape and its potential effects on individual health decisions. Toward that end, we conducted a survey of 600 adults in the state of Florida in order to better understand public attitudes and intentions regarding COVID-19 vaccination. In particular, we examined common objections to vaccination and whether vaccine hesitancy was correlated with exposure to misinformation about the origins and efficacy of COVID-19 vaccines. The survey results provide an important update for those health professionals currently fighting the COVID-19 pandemic (both on the clinical and informational fronts), while also contributing to our broader understanding of vaccine hesitancy as a sociomedical phenomenon. 


\section{METHODS}

\section{Survey Instrument and Administration}

The primary goals of this study were to better understand common objections to COVID-19 vaccination and the potential effects of misinformation on vaccine decisions. With these goals in mind, we conducted a cross-sectional, web-based survey of 600 adults in the state of Florida between June 3 and June 14, 2021. Florida is a relevant setting in which to study vaccine hesitancy due to surging COVID-19 cases and the political polarization of vaccine passports, mask mandates, and other prevention methods by Governor DeSantis, who has publicly opposed the Biden Administration's approach to COVID-19 prevention. ${ }^{11}$ Although an early frontrunner, Florida's vaccination rate has lagged behind other states and plateaued, ranking 9th at the time of this article. ${ }^{12,13}$ As of August 19, 2021, Florida is reported to account for $17.4 \%$ of new COVID-19 infections in the USA on an average daily basis. $^{14}$

Sample for the survey was purchased through an industryleading market research provider (Prodege MR), and survey respondents were selected using a stratified, quota sampling approach to ensure representativeness. Balanced quotas were determined (by region of the state) for gender, age, race, and ethnicity based on population estimates provided by the US Census Bureau and Florida's Office of Economic and Demographic Research. The sample of 600 represents a margin of error $+/-4$ for the state's population, and results are reported with a 95\% confidence level. Responses were required for all questions to ensure that missing response biases would not affect interpretation of the results.

Only those 18 and older were eligible to participate in the survey. While the sample is well representative of the state's adult population, some natural limitations accompany webbased panels. Most notably, the sampling method tends to be under-representative of those without a high-school degree, as well as residents of rural areas which may lack reliable internet service. The sample does not include residents of the state who are currently incarcerated, and while those residing in assisted care facilities are not excluded from participation, we suspect that they are under-represented given the administration method. These limitations should be kept in mind when interpreting the survey results. Table 1 provides a summary of the survey respondents in comparison to the state's demographics.

The questionnaire was designed by the authors to elicit feedback on several key issues, including (1) current vaccination status, (2) common objections to vaccination, and (3) exposure to vaccine-related misinformation. To ensure validity, the survey instrument was developed based on a thorough review of the most up-to-date public health guidance, professional media reporting, and national survey practices at the time. The instrument was pretested through the survey vendor's online platform and multiple quality control questions were included to ensure the highest quality of responses among participants. (Those
Table 1 Summary of Survey Respondents $(N=600)$

\begin{tabular}{|c|c|c|c|}
\hline & $\begin{array}{l}\text { USF } \\
\text { survey } \\
\text { sample }\end{array}$ & $\begin{array}{l}\text { Florida } \\
\text { demographicsa }\end{array}$ & $\begin{array}{l}p \text {-value (two- } \\
\text { proportion } z- \\
\text { test) }\end{array}$ \\
\hline \multicolumn{4}{|l|}{ Gender } \\
\hline Female & $52.0 \%$ & $51.1 \%$ & 0.659 \\
\hline Male & $48.0 \%$ & $48.9 \%$ & 0.659 \\
\hline \multicolumn{4}{|l|}{ Age } \\
\hline $18-24$ & $7.8 \%$ & $10.8 \%$ & $0.019 *$ \\
\hline $25-44$ & $30.2 \%$ & $31.2 \%$ & 0.585 \\
\hline $45-64$ & $33.8 \%$ & $32.4 \%$ & 0.453 \\
\hline $65+$ & $28.2 \%$ & $25.6 \%$ & 0.149 \\
\hline \multicolumn{4}{|l|}{ Race } \\
\hline Black/African & $17.7 \%$ & $16.9 \%$ & 0.616 \\
\hline American & & & \\
\hline White/ & $73.5 \%$ & $77.3 \%$ & $0.026^{*}$ \\
\hline \multicolumn{4}{|l|}{ Caucasian } \\
\hline Asian & $3.3 \%$ & $3.0 \%$ & 0.632 \\
\hline Pacific & $0.5 \%$ & $0.1 \%$ & $0.002 *$ \\
\hline \multicolumn{4}{|l|}{ Islander } \\
\hline American & $0.5 \%$ & $0.5 \%$ & 1.000 \\
\hline \multicolumn{4}{|l|}{ Indian/Alaska } \\
\hline \multicolumn{4}{|l|}{ Native } \\
\hline Other & $4.5 \%$ & $2.2 \%$ & $0.000 *$ \\
\hline \multicolumn{4}{|l|}{ Ethnicity } \\
\hline Hispanic & $25.0 \%$ & $26.4 \%$ & 0.436 \\
\hline Non-Hispanic & $75.0 \%$ & $73.6 \%$ & 0.436 \\
\hline \multicolumn{4}{|l|}{ Region } \\
\hline Panhandle & $6.7 \%$ & $7.2 \%$ & 0.613 \\
\hline Northeast & $12.7 \%$ & $12.4 \%$ & 0.843 \\
\hline \multicolumn{4}{|l|}{ Florida } \\
\hline Central & $25.3 \%$ & $25.5 \%$ & 0.925 \\
\hline \multicolumn{4}{|l|}{ Florida } \\
\hline West Coast & $27.2 \%$ & $21.9 \%$ & $0.002 *$ \\
\hline Southeast & $28.2 \%$ & $32.9 \%$ & $0.012 *$ \\
\hline Florida & & & \\
\hline
\end{tabular}

${ }^{a}$ Gender, race, ethnicity, and region quotas based on US Census Bureau's Population Estimates Program (PEP): https://www.census. gov/quickfacts/FL. Age quotas based on Florida Office of Economic and Demographic Research (EDR): http://edr.state.fl.us/Content/populationdemographics/data/index-floridaproducts.cfm ${ }^{*} p \leq 0.05$

who failed to correctly answer quality control questions were prevented from completing the survey and are not included in this analysis.)

In order to measure exposure to vaccine-related misinformation, we asked respondents whether they encountered each of eight common misinformation themes in the previous 6 months. Misinformation themes were identified based on an academic literature review, as well as current public health guidance. ${ }^{15-18}$ Understanding that it is impossible to measure all potential misinformation themes individually - as well as the need to mitigate respondent fatigue - we selected from this review a list of eight misinformation themes, which intentionally included medical misinformation as well as some political/conspiratorial misinformation themes. A majority of these themes have been identified by the CDC as being among the most critical falsehoods effecting vaccine hesitancy. ${ }^{15}$ While it is possible for respondents to have been exposed to additional misinformation themes not included in this analysis, the survey addressed those themes identified as most common in the academic literature and public health guidance, providing a strong proxy measure for overall misinformation 
exposure. (A copy of the survey instrument is provided in the Appendix to this report.)

\section{Statistical Methods}

The findings reported below are organized into three subsections, including (1) vaccination status, (2) common objections to vaccination, and (3) misinformation exposure, including its relationship with vaccine hesitancy. In the first subsection, we begin by examining differences in vaccination status across key demographic and political categories for all respondents $(n=600)$. This analysis includes a simple, bivariate examination of vaccination status across categories including age, gender, race, ethnicity, and political affiliation. We compare differences in vaccination status using chi-square $\left(\chi^{2}\right)$ tests with phi $(\varphi)$ tests providing a corresponding measure of association (IBM SPSS v.26, New York, NY).

Next, in order to examine key drivers of vaccine hesitancy, we focus on the subset of respondents who had not begun the vaccination process at the time that they completed the survey $(n=214)$. Among these respondents, those who indicated that they were unlikely to accept a vaccine $(n=97)$ were asked to share their objections to doing so. Response options for this question were determined based on public health guidance as well as professional media accounts, and respondents were given an opportunity to provide additional objections via an open-ended response. These responses were analyzed using simple descriptive statistics (IBM SPSS v.26, New York, NY).

Lastly, we examined exposure to common misinformation themes and its relationship to vaccination status among the initial sample $(n=600)$. Exposure to misinformation is reported using simple descriptive statistics. A principal components factor analysis ( $n=600$; varimax rotation) was attempted to determine whether the misinformation themes loaded into distinct categories (such as medical misinformation vs. political misinformation). The results did not support the existence of theoretically distinct categories, suggesting that exposure to misinformation among the survey respondents did not vary thematically. From there, using simple-chi-square tests, we examined the relationship between vaccination status and total misinformation exposure, as well as the individual relationships for each specific misinformation theme (IBM SPSS v.26, New York, NY).

As part of this analysis, we constructed a more comprehensive logit regression model to determine the effects of misinformation exposure on vaccination status while also accounting for demographic and political differences that are believed to influence vaccine hesitancy (STATA v.17, College Station, TX). The model was estimated (with robust standard errors) as follows:

$$
\begin{aligned}
\ln \left(\frac{\widehat{\pi}_{i}}{1-\widehat{\pi}_{i}}\right)= & \alpha \\
& +\sum[\beta(\text { demo })+\gamma(\text { edu })+\chi(\text { poli })+\theta(\text { pcp })+\rho(\text { expose })+\varepsilon]
\end{aligned}
$$

$\widehat{\pi}_{i}=\frac{\exp \alpha+\sum[\beta(\text { demo })+\gamma(\text { edu })+\chi(\text { poli })+\theta(\text { pcp })+\rho(\text { expose })+\varepsilon]}{1+\exp \alpha+\sum[\beta(\text { demo })+\gamma(\text { edu })+\chi(\text { poli })+\theta(\text { pcp })+\rho(\text { expose })+\varepsilon]}$

where $\widehat{\pi}_{i}$ is the predicted likelihood of a respondent being vaccinated against COVID-19; demo is a vector of demographic control variables (including age, gender, race, and ethnicity); edu is a measure of college education (where "no" is excluded as the reference category); poli is a measure of political affiliation (with "Democrat") excluded as the reference category; pcp is a binary measure of whether the respondent has spoken to their primary care provider about a COVID-19 vaccine (with "no" excluded as the reference category); and expose is a binary variable measuring exposure to misinformation (with "no" excluded as the reference category).

Results of the logit model are discussed below as odds ratios $\left(e^{\mathrm{b}}\right)$, which depict changes in the likelihood of a respondent being vaccinated based on a one unit increase in the independent variable. Odds ratios are multiplicative coefficients, such that ratios greater than 1 indicate an increase in the likelihood of vaccination, while ratios less than 1 indicate a decrease in the likelihood of vaccination. For ease of interpretation, odds ratios of less than 1 can be inverted so that $1 / e^{\mathrm{b}}$ represents the decreased likelihood of vaccination based on a one unit increase in the independent variable, ceteris paribus.

\section{FINDINGS}

A summary and analysis of the survey responses is provided below. The results are organized in subsections around the themes of (1) vaccination, (2) common objections, and (3) misinformation.

\section{Vaccination Among Survey Participants}

Among the survey respondents, $64.3 \%$ reported having received at least one dose of a COVID-19 vaccine, while $57.8 \%$ were "fully vaccinated" at the time of participation. Notable differences in vaccination rates were seen based on age as well as political affiliation (Table 2). Specifically, younger participants were significantly less likely to have been vaccinated. While $84 \%$ of those over 65 years of age reported receiving at least one COVID-19 vaccine dose, only $42.6 \%$ of those between 18 and 24 and $45.9 \%$ of those between 25 and 44 reported the same $\left(\chi^{2}=67.526 ; \varphi=0.335 ; p \leq 0.001\right)$.

In the case of political affiliation, self-identified Democrats were considerably more likely to report being vaccinated (73.4\%) than self-identified Republicans $(58.5 \%)$ and Independents $(56.5 \%)$. The observed difference was statistically significant where $\chi^{2}=16.334 ; \varphi=0.165 ; p \leq 0.001$. White respondents were more likely to be fully vaccinated than African Americans (66.4\% compared to 57.5\%), though this 
Table 2 Respondents Receiving at Least One Dose of a COVID-19 Vaccine By Population Groups $(n=600)$

\begin{tabular}{|c|c|c|}
\hline & \multicolumn{2}{|l|}{$n(\%)$} \\
\hline & Vaccinated & Unvaccinated \\
\hline \multicolumn{3}{|l|}{ Gendera } \\
\hline Female & $193(61.9)$ & $119(38.1)$ \\
\hline \multirow{2}{*}{\multicolumn{3}{|c|}{$A g e^{\mathrm{b}}$}} \\
\hline & & \\
\hline $18-24$ & $20(42.6)$ & $27(57.4)$ \\
\hline $25-44$ & $83(45.9)$ & $98(54.1)$ \\
\hline $45-64$ & $141(69.5)$ & $62(30.5)$ \\
\hline $65+$ & $142(84.0)$ & $27(16.0)$ \\
\hline \multicolumn{3}{|l|}{ Ethnicitya } \\
\hline Hispanic & $96(64.0)$ & $54(36.0)$ \\
\hline Non-Hispanic & $290(64.6)$ & $160(35.6)$ \\
\hline \multicolumn{3}{|l|}{ Racea } \\
\hline African American & $61(57.5)$ & $45(42.5)$ \\
\hline White & $293(66.4)$ & $148(33.6)$ \\
\hline Other & $32(60.4)$ & $21(39.6)$ \\
\hline \multicolumn{3}{|l|}{ Political affiliation ${ }^{\mathrm{c}}$} \\
\hline Democrat & $190(73.4)$ & $69(26.6)$ \\
\hline Independent & $100(56.5)$ & $77(43.5)$ \\
\hline Republican & $96(58.5)$ & $68(41.5)$ \\
\hline
\end{tabular}

${ }^{a}$ Not statistically significant at 0.05 level. ${ }^{b} \chi^{2}=67.526 ; \varphi=0.335 ; p \leq$ $0.001 ;{ }^{c} \chi^{2}=16.334 ; \varphi=0.165 ; p \leq 0.001$.

difference was not statistically significant. While also not statistically significant, slightly higher levels of vaccination were observed among males (67.0\% compared to $61.9 \%$ of females). In each case, the differences were consistent with those observed in prior studies of vaccine intent. ${ }^{6,19}$

\section{Common Objections to Vaccination}

Among those participants who had not begun the vaccination process at the time of the survey $(n=214)$, just under half $(45.3 \%)$ said that they would either "probably not" or "definitely not" get vaccinated. Among those who indicated that they were unlikely to accept a vaccine $(n=97)$, two particular objections were noted by a majority of respondents, including concerns over the potential side effects of the vaccine (74.2\%) and fears that the vaccines were created too quickly to be adequately tested $(50.5 \%)$ (Figure 1). Another fifth of these respondents $(20.6 \%)$ indicated that they don't believe the vaccines are effective against COVID-19, while the same number $(20.6 \%)$ said they were simply not concerned about contracting COVID-19.

Respondents were provided an opportunity to share additional objections to vaccination via an open-ended response option. Only 5 respondents $(5.1 \%)$ opted to provide an openended response. Of these, three respondents reiterated concerns about the speed of the vaccine's development and lack of available data on long-term effects, while two reiterated that they don't view COVID-19 as a serious threat. The openended responses did not yield additional objections beyond those provided in the initial response options.

\section{Misinformation Exposure and Vaccination}

Table 3 summarizes the frequency with which respondents reported exposure to eight specific misinformation themes.
According to the survey results, exposure to misinformation has been widespread, with nearly three-quarters of respondents (73.2\%) reporting some degree of misinformation exposure in the referenced time period. The most commonly identified misinformation themes included claims that COVID-19 vaccines contain a "live strain" of the virus (42.8\%), COVID19 vaccines contain $5 \mathrm{G}$ microchips (38.5\%), COVID-19 vaccines modify people's genes and alter their DNA (36.3\%), and that vaccination would be mandated by the CDC (36.3\%).

We observed a statistically significant correlation between vaccination status and aggregate exposure to misinformation $\left(\chi^{2}=11.349 ; \varphi=0.138 ; p \leq 0.05\right)$. Among those who did not report any exposure to misinformation, $73.8 \%$ of respondents were vaccinated (Table 4 ). That number fell to $62.9 \%$ when respondents were exposed to just one misinformation theme. For those who reported exposure to six or more misinformation themes, the vaccination rate fell to $52.2 \%$. When considering these results, it should be noted that the cross-sectional nature of the data does not allow for a time-ordered observation of events.

The results presented in Table 5 provide a more nuanced look at the individual relationship between each specific misinformation theme and vaccination status. For seven of the eight misinformation themes, vaccination rates were higher among those who were not exposed to the false stories/claims. (They were slightly lower in the case of the $5 \mathrm{G}$ microchip story, though this difference was not statistically significant.) Among the remaining seven themes, the observed differences were found to be statistically significant in four instances. These include the claim that COVID-19 vaccines contain a "live strain" of the virus $\left(\chi^{2}=4.515 ; \varphi=0.034 ; p \leq 0.05\right)$, that COVID-19 vaccines were designed to reduce the world's population $\left(\chi^{2}=11.244 ; \varphi=-0.137 ; p \leq 0.001\right)$, that COVID-19 was created to increase vaccine sales $\left(\chi^{2}=\right.$ $14.881 ; \varphi=-0.157 ; p \leq 0.001)$, and that the daughter of the Russian president died from a COVID-19 vaccine $\left(\chi^{2}=6.602\right.$; $\varphi=-0.105 ; p \leq 0.05$ ).

The most substantial effect sizes were seen in the latter two cases, where vaccination rates were $20 \%$ higher among those who were not exposed to these misinformation themes. Notably, three of the four statistically significant themes were the least commonly encountered and contained political/ conspiratorial misinformation. In contrast, only one of the statistically significant themes (the "live strain" claim) represented medical misinformation; this was the most commonly encountered theme of those included in this survey.

Lastly, Table 6 summarizes results from the logistic regression model, which examines the effect of several factors on vaccination status, including misinformation exposure. The results show that exposure to misinformation is associated with a lower likelihood of vaccination, even after accounting for other predictors of vaccine hesitancy. Those exposed to at least one misinformation theme were 1.577 times $\left(1 / e^{\mathrm{b}}\right)$ less likely to be vaccinated, ceteris paribus. A disaggregated regression model considering each individual misinformation 


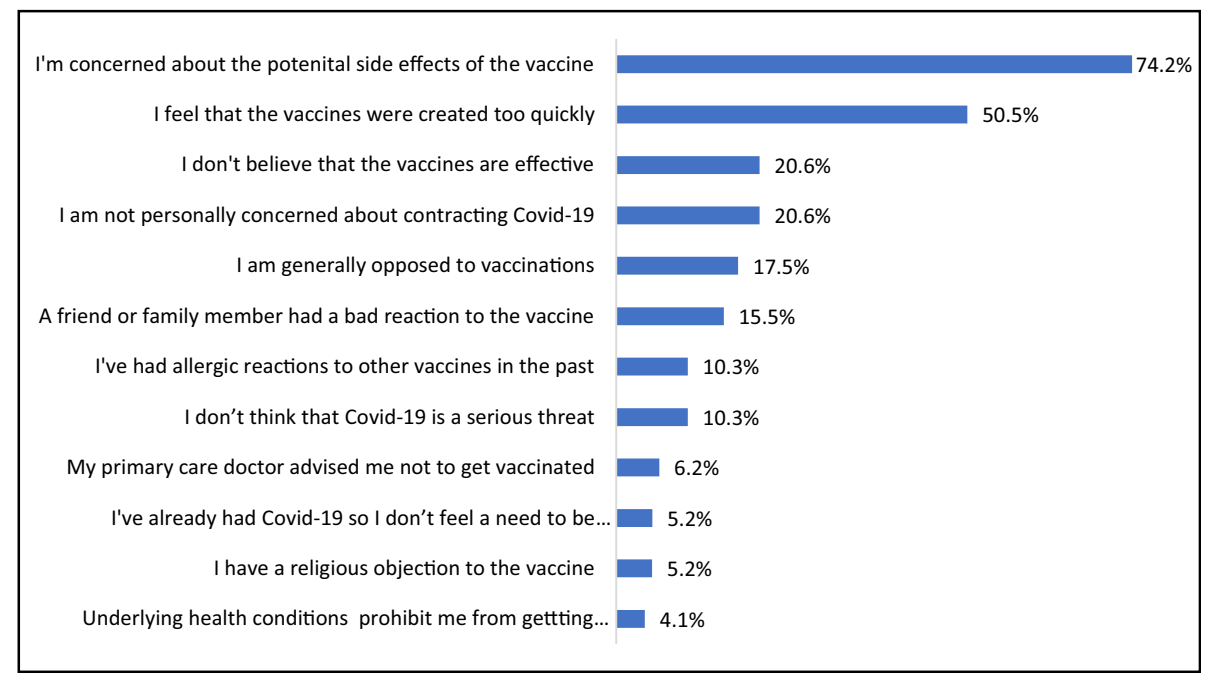

Figure 1 Common objections to vaccination among those who will "probably not" or "definitely not" get vaccinated ( $n=97)$. Note: Question was only posed to those who say they will either "probably not" or "definitely not get vaccinated"; $n=97$

theme was also tested. The overall results were unchanged, though only one specific misinformation theme (re: increasing vaccine sales) was associated with a statistically significant decline in the likelihood of vaccination.

Other significant predictors of vaccination status included age, education, and political affiliation. Those with a 4-year college degree or higher were 2.153 times more likely to be vaccinated, while those over 65 years of age were 6.250 times more likely to be vaccinated than those between the ages of 18 and 24. In contrast, self-identified Republicans were 3.356 times $\left(1 / e^{\mathrm{b}}\right)$ less likely to vaccinated than self-identified Democrats, while political Independents were 2.037 times less likely. Gender, race, and ethnicity were not significant predictors of vaccination status, ceteris paribus, nor were conversations with primary care providers. (Note that only $32.3 \%$ of respondents reported having a conversation about COVID-19 vaccination with their primary care provider.)

Table 3 Frequency of Exposure to Vaccine-Related Misinformation Themes $(n=600)$

\begin{tabular}{lll}
\hline \hline $\begin{array}{l}\text { In the past 6 months, have you read or } \\
\text { heard any of the following stories/claims } \\
\text { about COVID-19 vaccines }\end{array}$ & Frequency & Percent \\
\hline $\begin{array}{l}\text { COVID-19 vaccines contain a "live strain" of } \\
\text { the virus }\end{array}$ & 257 & 42.8 \\
$\begin{array}{l}\text { COVID-19 vaccines contain 5G microchips } \\
\text { COVID-19 vaccines will be mandated by the }\end{array}$ & 231 & 38.5 \\
$\begin{array}{l}\text { CDC } \\
\text { COVID-19 vaccines modify people's genes }\end{array}$ & 218 & 36.3 \\
$\begin{array}{l}\text { and alter their DNA } \\
\text { COVID-19 vaccines may cause infertility }\end{array}$ & 207 & 36.3 \\
$\begin{array}{l}\text { COVID-19 vaccines were designed to reduce } \\
\text { the world's population }\end{array}$ & 182 & 34.5 \\
$\begin{array}{l}\text { COVID-19 vaccines were created before the } \\
\text { pandemic started in order to increase vaccine }\end{array}$ & 113 & 18.3 \\
sales & & \\
$\begin{array}{l}\text { The daughter of the Russian president died } \\
\text { from the COVID-19 vaccine }\end{array}$ & 36 & 6.0 \\
$\begin{array}{l}\text { Did not report any misinformation exposure } \\
\text { n=600 }\end{array}$ & 161 & 26.8 \\
\hline
\end{tabular}

\section{DISCUSSION}

In the pre-pandemic era, the World Health Organization (WHO) labeled vaccine hesitancy as one of the "top ten threats to global health" in $2019 .{ }^{20}$ Despite being one of the most effective methods for infectious disease control, Americans have become increasingly skeptical of vaccinations, with vaccine rates declining notably in the past decade. ${ }^{21}$ Factors such as a previous study correlating MMR vaccination with autism, and the misinformation which followed, may have played a role in this trend..$^{22}$ Our study found that only $57.8 \%$ of survey respondents were fully vaccinated at the time of their participation in this survey. Herd immunity vaccination percentage requirements for COVID-19 in a population is an area of emerging research; however, the observed $57.8 \%$ is far less than required for herd immunity in diseases such as measles $(95 \%)$ and polio (80\%) according to the WHO. Furthermore, the WHO considers herd immunity by pathogen exposure unethical due to potential suffering and mortality. ${ }^{23}$

Understanding the reasoning behind vaccine hesitancy is an important step in effectively targeting public health messaging. Our survey found that the three most common reasons that respondents give for holding off on receiving the COVID-19 vaccine are (1) the potential for side effects, (2) the speed at which the vaccine was created, and (3) surprisingly, a lack of confidence in the vaccine's effectiveness. While the potential for side effects is a reasonable concern, the benefits of

Table 4 Misinformation exposure by vaccination status $(n=600)$

\begin{tabular}{llllll}
\hline \hline Vaccinated & \multicolumn{4}{l}{$\begin{array}{l}\text { Total reported exposure to misinformation themes } \\
\text { (as \% of column total) }\end{array}$} \\
\cline { 2 - 6 } & $\begin{array}{l}\text { No } \\
\text { reported } \\
\text { exposure }\end{array}$ & $\begin{array}{l}\text { One } \\
\text { theme }\end{array}$ & $\begin{array}{l}\text { Two- } \\
\text { three } \\
\text { themes }\end{array}$ & $\begin{array}{l}\text { Four- } \\
\text { five } \\
\text { themes }\end{array}$ & $\begin{array}{l}\text { Six- } \\
\text { eight } \\
\text { themes }\end{array}$ \\
\hline Yes & 73.8 & 62.9 & 61.0 & 64.3 & 52.2 \\
No & 26.3 & 37.1 & 39.0 & 35.7 & 47.8 \\
\hline 2 & & $0.138 ;$ & 0.023 & &
\end{tabular}


Table 5 Percent vaccinated based by exposure to specific misinformation themes $(n=600)$

\begin{tabular}{|c|c|c|}
\hline \multirow{2}{*}{$\begin{array}{l}\text { In the past } 6 \text { months, have you read or } \\
\text { heard any of the following stories/claims } \\
\text { about COVID-19 vaccines }\end{array}$} & \multicolumn{2}{|c|}{$\%$ vaccinated } \\
\hline & Exposed & $\begin{array}{l}\text { Not } \\
\text { exposed }\end{array}$ \\
\hline $\begin{array}{l}\text { COVID-19 vaccines contain a "live strain" } \\
\text { of the virus }\end{array}$ & $59.5^{\mathrm{a}}$ & $67.9^{\mathrm{a}}$ \\
\hline COVID-19 vaccines contain $5 \mathrm{G}$ microchips & 66.2 & 63.1 \\
\hline $\begin{array}{l}\text { COVID-19 vaccines will be mandated by the } \\
\text { CDC }\end{array}$ & 60.1 & 66.8 \\
\hline $\begin{array}{l}\text { COVID-19 vaccines modify people's genes } \\
\text { and alter their DNA }\end{array}$ & 61.9 & 65.7 \\
\hline COVID-19 vaccines may cause infertility & 61.4 & 65.9 \\
\hline $\begin{array}{l}\text { COVID-19 vaccines were designed to reduce } \\
\text { the world's population }\end{array}$ & $54.4^{\mathrm{b}}$ & $68.7^{b}$ \\
\hline $\begin{array}{l}\text { COVID-19 vaccines were created before the } \\
\text { pandemic started in order to increase vaccine } \\
\text { sales }\end{array}$ & $48.7^{\mathrm{c}}$ & $68.0^{\mathrm{c}}$ \\
\hline $\begin{array}{l}\text { The daughter of the Russian president died } \\
\text { from the COVID-19 vaccine }\end{array}$ & $44.4^{\mathrm{d}}$ & $65.6^{\mathrm{d}}$ \\
\hline
\end{tabular}

COVID-19 vaccines have shown to be greater than the potential risks. Furthermore, despite the speed at which the vaccine was developed, the Center for Disease and Control notes that the safety monitoring of the COVID-19 vaccine development was "the most intense safety monitoring in U.S. history". ${ }^{24}$ The findings suggest that amplifying these messages will continue to be an important task for public health professionals moving forward.

Interestingly, $20.6 \%$ of the survey respondents felt that vaccines were not effective despite the positive clinical trial results and "real world" research to support their efficacy. ${ }^{25}$ It is not clear whether this belief itself is related to misinformation; however, it does indicate that current methods of public health messaging regarding vaccination efficacy may be inadequate. Given that $24.3 \%$ of unvaccinated respondents in this

Table 6 Logistic regression: Odds that vaccination status = "Yes" $(n=600)$

\begin{tabular}{llll}
\hline \hline & $\boldsymbol{e}^{\mathbf{b}}$ & $\boldsymbol{\beta}$ & s.e. \\
\hline Age & & & \\
18-24 (ref cat) & - & - & - \\
25-44 & 0.788 & -0.237 & 0.298 \\
45-64 & 2.733 & 1.005 & 1.022 \\
65+ & 6.250 & 1.833 & 2.542 \\
Gender (male) & 1.196 & 0.179 & 0.235 \\
Hispanic (yes) & 1.121 & 0.114 & 0.267 \\
Race & & & \\
$\quad$ African American (ref cat) & - & - & - \\
$\quad$ White & 1.559 & 0.444 & 0.425 \\
$\quad$ Other & 1.081 & -0.078 & 0413 \\
College degree (yes) & 2.153 & 0.767 & 0.467 \\
Political affiliation & & & \\
$\quad$ Democrat (ref cat) & - & - & - \\
Independent/other & 0.491 & -0.711 & 0.119 \\
Republican & 0.298 & -1.209 & 0.073 \\
Spoken to primary care provider (yes) & 1.377 & 0.291 & 0.319 \\
Exposure to misinformation (yes) & 0.634 & -0.456 & 0.140 \\
Constant & 0.981 & -0.019 & 0.422 \\
-2LL & -330.582 & - & - \\
Pseudo $R^{2}$ & 0.154 & - & - \\
\hline
\end{tabular}

$* p \leq 0.05 ; * * p \leq 0.001$ study indicated they were unsure whether they would undergo vaccination, increasing public health messaging efforts to reach this portion of the population who are "open" to considering vaccination should be paramount.

Our findings also suggest that vaccine hesitancy may be driven in large part by the increasing politicization of public health policy, which appears to have reached its zenith in the case of COVID-19. ${ }^{7,26}$ Our survey results highlighted a significant difference in the rate of vaccination based on political affiliation, with nearly three-quarters of self-identified Democrats being vaccinated (73.4\%), compared with less than twothirds of Republicans $(58.5 \%)$ and political Independents (56.5\%). Even when accounting for additional demographic factors and misinformation exposure, political affiliation was a very strong predictor of vaccination. This presents a unique challenge for public health messaging, as politicization makes it more likely that consumers will seek informational cues from political thought leaders rather than health professionals.

Furthermore, our findings affirm that widespread exposure to misinformation is a barrier to consumer health education on the vaccine and its benefits. In the survey results, those health information consumers with more exposure to misinformation regarding the COVID-19 vaccine were less likely to be vaccinated. Furthermore, the rate of vaccination among respondents continued to decline with exposure to two or more misinformation themes. Although the timing of misinformation exposure could not be determined by the survey, the results suggest that misinformation may play a very important role in vaccination status. This conclusion is consistent with other research on COVID-19 vaccine hesitancy. For example, a recent study found a $6.4 \%$ point decline in vaccination with exposure to misinformation in the USA and a $6.2 \%$ point decline in the UK. ${ }^{27}$

Of particular concern is the proliferation of political and conspiratorial misinformation, which we found to have the most substantial relationship to vaccine hesitancy. In some cases, we found a $20 \%$ lower rate of vaccination among those exposed to specific conspiratorial claims, such as the COVID19 virus being created to increase vaccine sales and the Russian president's daughter dying from a COVID-19 vaccine. The strong relationship between these claims and vaccine hesitancy further underscores the effects of politicization on the COVID-19 pandemic (and public health efforts in general). The tendency of conspiratorial claims to be circulated and amplified among closed circles of like-minded partisans means that these types of misinformation are often difficult for public health officials to identify, and even more difficult to combat. ${ }^{28}$ The documented propensity of information consumers to self-select partisan media sources ${ }^{29,30}$ is believed to result in political echo chambers wherein these types of misinformation are easily amplified. ${ }^{31}$ Strategic and targeted messaging will be essential in order to increase vaccine acceptance among individuals exposed to these types of misinformation.

With these findings in mind, we note that public health officials and healthcare providers should remain diligent in 
their efforts to identify and understand evolving objections, both rational and misinformed, that patients have about the COVID-19 vaccine. To address patient concerns about vaccine safety, they may opt to facilitate open discussions about vaccine fears through community outreach efforts or via social media and other outlets. Research has shown that acknowledging fears regarding difficult issues can promote trust in the messenger of the information. ${ }^{32}$ Trust in public health messaging is especially important during public health emergencies when information is dynamic and rapidly evolving. Because political affiliation is so strongly correlated with vaccine hesitancy, this means that utilizing political figures to promote reliable information may be an essential means of increasing vaccination rates within those ideological groups.

In this instance, targeted and consistent messaging from Republican leaders in particular will be necessary to overcome politicized vaccine hesitancy. ${ }^{33}$ While reporting shows that many Republican political leaders have been vaccinated against COVID-19, they have thus far been less inclined to promote vaccination among their political adherents. Recent attempts to promote vaccination on the part of Republican thought leaders such as Sean Hannity and Senate Minority Leader Mitch McConnell have been a positive step, ${ }^{34}$ though these messages have been somewhat muted by simultaneous rhetoric and efforts against vaccine and mask mandates in various high-risk settings. ${ }^{35}$ More consistent messaging on the part of Republican leaders will be necessary to ensure increased rates of vaccine acceptance. Public health officials can help to facilitate these efforts by partnering with willing political leaders at the local, state, and national levels.

Another potential strategy may include encouraging hesitant patients to reengage with their primary care providers, who are well-positioned to communicate reliable vaccinerelated information. Recent studies have found personal appeals from practicing physicians to be more effective than institutional communications for promoting public health guidance. ${ }^{36}$ However, only $32.3 \%$ of respondents to this survey reported having a conversation with their own primary care provider about COVID-19 vaccination. Among other factors, the politicization of the COVID-19 pandemic has resulted in increased reliance on "political cues" on the part of many information consumers, at the expense of conversations that would typically be sought with one's most trusted healthcare providers. A proactive campaign on the part of public health officials and political leaders to direct patients back toward these provider-patient conversations may help to overcome some cases of vaccine hesitancy.

Lastly, we note that the increased role of social media in health information seeking has likely also played a significant role in the widespread exposure to misinformation observed in our study. Evidence from recent studies show that health consumers have relied heavily on social media to learn and stay informed about the COVID-19 pandemic. ${ }^{10,37}$ Studies have also found alarming rates of misinformation about COVID-19- both medical and political - being circulated on social media platforms such as Facebook and Twitter. ${ }^{38,39}$ In order to counteract these trends, public health professionals will need to become increasingly savvy in their use of social media to anticipate, identify, and respond to health-related misinformation. Preliminary evidence suggests that such interventions may be effective. For example, one study found that corrective infographics created by the World Health Organization were effective in reducing scientific misperceptions about COVID-19 prevention. ${ }^{40}$ In another study from early 2021, those engaging with more credible, scientific sources on social media reported a greater likelihood of undergoing vaccination. ${ }^{10}$ Expanding social media presence on the part of both public health organizations and individual practitioners may help at least in part to offset the rapid spread of misinformation associated with social networking sites.

\section{LIMITATIONS AND CONCLUSION}

While our results shed light on several important issues in regard to vaccine hesitancy and its relationship to misinformation, there are important limitations to keep in mind when interpreting these results, as well as several opportunities for additional research. As noted above, the sample of respondents included in this analysis may have under-represented some high-riskand/or important groups, including (1) those under 18 years of age who are eligible for vaccination, (2) those lacking a high school education (note that education was found to be significantly related to vaccination rates), (3) residents of nursing homes and correctional facilities, and (4) rural residents who lack reliable internet service. Additionally, our survey focused narrowly on residents of Florida; a national replication of this analysis may yield important additional insights into vaccine hesitancy and misinformation in the US context.

Additionally, we rely on a proxy measure for overall misinformation exposure which is constructed from eight specific misinformation themes. While these represent some of the most commonly encountered misinformation themes (according to the $\mathrm{CDC}$ ), it is likely that information consumers encounter a host of additional misinformation depending on their patterns of news and information consumption. Our findings suggest that exposure to political/conspiratorial misinformation themes in particular is highly correlated with vaccine hesitancy. Additional research might consider the breadth and nature of these themes, particularly those not captured in this analysis. We also note that the lack of timeordered observation in our study prevents us from fully understanding causation and the potential for simultaneity in our findings. Additional research is needed in order to understand the extent to which misinformation exposure precedes vaccine hesitancy vs. the extent to which it simply reflects participation in politicized information environments. Measuring the information sources that consumers rely on may also yield important insights into these critical relationships. 
Despite these limitations, our survey results do contribute to previous research on misinformation and vaccine hesitancy by quantifying exposure to specific misinformation themes. Understanding the volume and nature of misinformation themes the public is exposed to regarding COVID-19 vaccines may aid public health officials in targeting vaccine messaging to more directly address reasons for vaccine hesitancy and allay fears. More research is needed on how to adequately address vaccine misinformation, especially with regard to timing and platform/methods, in order to improve trust in public health messengers, which may ultimately aid in reducing vaccine hesitancy. Our findings also highlight the potentially deleterious effects of politicization on public health outcomes. While public health organizations and professionals may — for good reason - be reluctant to engage in political matters, it appears increasingly necessary for health professionals to understand the causes and effects of politicization in public health issues and to develop strategies for overcoming these obstacles. By better understanding these root causes of vaccine hesitancy including the specific misinformation themes that induce it health professionals can more effectively promote vaccination and expand public confidence in proven medical advances.

Corresponding Author: Stephen R. Neely, PhD; School of Public Affairs, University of South Florida, 4202 E. Fowler Ave, SOC 107, Tampa, FL 33620, USA (e-mail: srneely@usf.edu).

Supplementary Information The online version contains supplementary material available at https://doi.org/10.1007/s11606-02107171-z.

\section{Declarations:}

Conflict of Interest: The authors report no conflicts of interest, financial or otherwise, related to this study or submission.

Institutional Review: This study is deemed exempt by the authors' Institutional Review Board (IRB). A de-identified data set is purchased through sample vendor, so the authors do not have any human subjects interactions in fielding the survey.

\section{REFERENCES}

1. Daly M, Jones A, Robinson E. Public trust and willingness to vaccinate against COVID-19 in the US from October 14, 2020 to March 29, 2021. JAMA 2021; 325(23): 2397-2399.

2. Rief W. Fear of adverse effects and COVID-19 vaccine hesitancy: Recommendations of the treatment expectation expert group. JAMA Health Forum 2021; 2(4): e210804.

3. Centers for Disease Control and Prevention. COVID-19 vaccinations in the United States. Available at https://COVID.cdc.gov/COVID-datatracker/\#vaccinations Accessed June 18, 2021.

4. Erman M, Shalal A. Biden, Harris urge vaccinations as U.S. looks likely to miss July 4 target. Reuters, June 18, 2021. Available at https://www. reuters.com/world/us/us-has-administered-300-million-COVID-19shots-150-days-white-house-official-2021-06-18/ Accessed June 19, 2021

5. Reitsma, M, Artiga S, Goldhaber-Fiebert J, Joseph N, Kates J, Levitt L, Rouw A, Salomon J. Disparities in reaching COVID-19 vaccination rates by race/ethnicity as of July 4 . Kaiser Family Foundation, June 14, 2021. Available at https://www.kff.org/racial-equity-and-health-policy/
issue-brief/disparities-in-reaching-COVID-19-vaccination-benchmarksprojected-vaccination-rates-by-race-ethnicity-as-of-july-4/ Accessed on June 19, 2021.

6. Troiano G, Nardi A. Vaccine hesitancy in the era of COVID-19. Public Health 194: 245-251..

7. Wood S, Schulman K. Beyond politics - promoting COVID-19 vaccination in the United States. New England Journal of Medicine 2021; 384(7): e23(1)-e23(8).

8. Charron J, Gautier A, Jestin C. Influence of information sources on vaccine hesitancy and practices. Medecine et maladies infectieuses 2020; 50: 727-733.

9. Loomba S, de Figueiredo A, Piatek SJ, de Graaf $\mathbf{K}$, Larson HJ. Measuring the impact of COVID-19 vaccine misinformation on vaccination intent in the UK and USA. Nature Human Behavior 2020; 5(3): 337348.

10. Neely S, Eldredge C, Sanders R. Health Information Seeking Behaviors on Social Media During the COVID-19 Pandemic Among American Social Networking Site Users: Survey Study. Journal of Medical Internet Research 2021;23(6):e29802.

11. Vlamis K. Florida Gov. Ron DeSantis snapped at Biden for criticizing his handling of COVID-19: 'Why don't you do your job?' Business Insider, August 4, 2021. Available at https://www.businessinsider.com/floridaron-desantis-snaps-at-biden-covid-19-criticism-2021-8? web= $1 \& w d L O R=c 6 B 6 B 7 B 44-77 D E-4 B 36-8 A 76-84 D C 18 B D 5 B E D$ Accessed August 13, 2021

12. Centers for Disease Control and Prevention. COVID Data Tracker. June 14, 2021. Available at https://covid.cdc.gov/covid-data-tracker/ \#datatracker-home Accessed August 13, 2021.

13. Florida Department of Health. COVID-19 Weekly Situation Report: June 4, 2021-June 10, 2021. Available at http://ww11.doh.state.fl.us / comm/_partners/covid19_report_archive/covid19-data/covid19_data_ 20210611.pdf Accessed August 13, 2021

14. New York Times. Coronavirus in the U.S.: Latest map and case count. Updated August 19, 2021. Available at https://www.nytimes.com/ interactive/2021/us/covid-cases.html Accessed August 19, 2021.

15. Centers for Disease Control and Prevention. Myths and facts about COVID-19 vaccines. Updated June 17, 2021. Available at https://www. cdc.gov/coronavirus/2019-ncov/vaccines/facts.html Accessed June 19, 2021.

16. Cleveland Clinic. Common COVID-19 vaccine myths explained. December 23, 2020. Available at https://health.clevelandclinic.org/commonCOVID-19-vaccine-myths-explained/ Accessed June 19, 2021.

17. Hotez P, Batista C, Ergonul O,... Bottazzi ME. Correcting COVID-19 vaccine misinformation. EClinical Medicine 2021; 33:100780.

18. Islam MS, Kamal AHM, Kabir A, ... Seale H. COVID- 19 vaccine rumors and conspiracy theories: The need for cognitive inoculation against misinformation to improve vaccine adherence. PLoS One 2021; 16(5): 117. PMID: 33718854

19. Neely, S. Taking Americas temperature on the vaccine: COVID-19 vaccine and policy survey. Florida Center for Cybersecurity: January 14, 2021. https://cyberflorida.org/report/vaccine-and-policy/

20. Geoghegan S, O'Callaghan KP, Offit PA. Vaccine safety: Myths and misinformation. Frontiers in Microbiology 2020; 11: 372.

21. McClure CC, Cataldi JR, O'Leary ST. Vaccine hesitancy: Where we are and where we are going. Clinical Therapeutics 2017; 39(8): 1550-1562. Available at https://www.clinicaltherapeutics.com/action/showPdf? pii=S0149-2918\%2817\%2930770-1

22. Davidson M. Vaccination as a cause of autism - myths and controversies. Dialogues in Clinical Neuroscience 2017; 19(4): 403-407.

23. World Health Organization. Coronavirus disease (COVID-19): Herd immunity, lockdowns, and COVID-19. December 30, 2020. Available at https://www.who.int/news-room/q-a-detail/herd-immunity-lockdownsand - covid - 19 ? g c lid = C jw KCAjwie u G BhAs EiwA 1 Ly_ nbBrneuoXLeUFtOgARFiBFMavNjcDilx8BGBvSH85NmyT7fCnZ21hoClXkQAvD_BwE Accessed July 1, 2021.

24. Centers for Disease Control. Safety of COVID-19 vaccines. June 28, 2021. Available at https://www.cdc.gov/coronavirus/2019-ncov/vaccines/safety/safety-of-vaccines.html Accessed July 1, 2021.

25. Centers for Disease Control. COVID-19 vaccines work. May 20, 2021. Available at https://www.cdc.gov/coronavirus/2019-ncov/vaccines/effectiveness/work.html Accessed July 1, 2021.

26. Neely S. COVID-19: Social media, politicization, and misinformation. Florida Center for Cybersecurity: January 25, 2021. https://cyberflorida. org/report/COVID-social-media/

27. Loomba S, de Figueiredo A, Piatek SJ, de Graaf K, Larson HJ. Measuring the impact of COVID-19 vaccine misinformation on vaccination intent in 
the UK and USA. Nature Human Behavior 2021; 5: 337-348. https://doi. org/10.1038/s41562-021-01056-1

28. Cinelli M., Quattrociocchi W., Galeazzi A. et al. The COVID-19 social media infodemic. Sci Rep 2020; 10, 16598. https://doi.org/10.1038/ s41598-020-73510-5

29. Hahn KS, Ryu, S. Park S. Fragmentation in the Twitter Following of News Outlets: The Representation of South Korean Users "Ideological and Generational Cleavage". Journalism and Mass Communication Quarterly 2015; 92(1): 56-76.

30. Iyengar S. and Hahn KS. "Red Media, Blue Media”: Evidence of Ideological Selectivity in Media Use. Journal of Communication 2009; 59(1): 19-39.

31. Sunstein CR. 2017. Republic: Divided Democracy in the Age of Social Media. Princeton University Press: Princeton, NJ.

32. Public Health Institute. Communicating about the COVID-19 vaccines: Guidance and sample messages for public health practitioners. May 5, 2021. Available at https://www.phi.org/thought-leadership/communicating-about-the-covid-19-vaccines-guidance-and-sample-messages-forpublic-health-practitioners/ Accessed June 30, 2021.

33. Pink SL, Chu J, Druckman JN, Rand DG, Willer R. Elite party cues increase vaccination intentions among Republicans. Proceedings of the National Academy of Sciences Aug 2021, 118 (32) e2106559118; DOI https://doi.org/10.1073/pnas.2106559118

34. Morgan D. McConnell aims to boost U.S. Republican vaccination rate by countering 'bad advice'. Reuters, July 28, 2021. Available at https:// www.reuters.com/world/us/mcconnell-strives-counter-bad-adviceboost-us-republican-vaccination-rate-2021-07-28/ Accessed August 13, 2021.
35. Bauder D. Conservative media offers mixed messages on COVID-19 vaccine. Associated Press, July 21, 2021. Available at https://apnews. com/article/joe-biden-health-government-and-politics-arts-and-entertainment-media-74f36fead348661aeba170422381a972 Accessed August 13, 2021.

36. Solnick RE, Chao G, Ross RD, Kraft-Todd GT, Kocher KE. Emergency physicians and personal narratives improve the perceived effectiveness of COVID-19 public health recommendations on social media: A randomized experiment. Acad Emerg Med 2021 Feb;28(2):172-183

37. Stjernswärd S, Ivert AK, Glasdam S. Perceptions and effects of COVID-19 related information in Denmark and Sweden - a web-based survey about COVID-19 and social media. Z Gesundh Wiss 2021 26:1-15.

38. Kouzy R, Jaoude JA, Kraitem A, El Alam MB, Karam B, Adib E, et al Coronavirus goes viral: Quantifying the COVID-19 misinformation epidemic on Twitter. Cureus 2020;12(3):e7255.

39. Managing the COVID-19 infodemic: Promoting healthy behaviours and mitigating the harm from misinformation and disinformation. World Health Organization. 2020 Sep 23. URL: https://tinyurl.com/k4zrca9e

40. Vraga EK, Bode L. Addressing COVID-19 misinformation on social media preemptively and responsively. Emerg Infect Dis 2021;27(2):396-403.

Publisher's Note: Springer Nature remains neutral with regard to jurisdictional claims in published maps and institutional affiliations. 